\title{
Review of: "Preclinical evidence of remote ischemic conditioning in ischemic stroke, a metanalysis update"
}

Norbert Nighoghossian ${ }^{1}$

1 Centre Hospitalier Universitaire de Lyon

Potential competing interests: The author(s) declared that no potential competing interests exist.

THis is a relevant and useful review of the potential value of remote conditionning that needs further investigation in ischemic stroke in the context of thrombectomy. I approve the manuscript clearly 